\title{
Accuracy and Sources of Error for an Angle Independent Volume Flow Estimator
}

\author{
Jensen, Jonas; Olesen, Jacob Bjerring; Hansen, Peter Møller; Nielsen, Michael Bachmann; Jensen, \\ Jørgen Arendt
}

\section{Published in:}

Proceedings of IEEE International Ultrasonics Symposium

Link to article, DOI:

10.1109/ULTSYM.2014.0425

Publication date:

2014

Link back to DTU Orbit

\section{Citation (APA):}

Jensen, J., Olesen, J. B., Hansen, P. M., Nielsen, M. B., \& Jensen, J. A. (2014). Accuracy and Sources of Error for an Angle Independent Volume Flow Estimator. In Proceedings of IEEE International Ultrasonics Symposium (pp. 1714-1717). IEEE. https://doi.org/10.1109/ULTSYM.2014.0425

\section{General rights}

Copyright and moral rights for the publications made accessible in the public portal are retained by the authors and/or other copyright owners and it is a condition of accessing publications that users recognise and abide by the legal requirements associated with these rights.

- Users may download and print one copy of any publication from the public portal for the purpose of private study or research.

- You may not further distribute the material or use it for any profit-making activity or commercial gain

- You may freely distribute the URL identifying the publication in the public portal 


\title{
Accuracy and Sources of Error for an Angle Independent Volume Flow Estimator
}

\author{
Jonas Jensen ${ }^{1}$, Jacob Bjerring Olesen ${ }^{1}$, Peter Møller Hansen ${ }^{1,2}$, Michael Bachmann Nielsen ${ }^{2}$ and Jørgen Arendt Jensen ${ }^{1}$ \\ ${ }^{1}$ Center for Fast Ultrasound Imaging, Dept. of Elec. Eng. Bldg. 349, \\ Technical University of Denmark, DK-2800 Lyngby, Denmark \\ ${ }^{2}$ Department of Radiology, Copenhagen University Hospital, DK-2100 Copenhagen, Denmark.
}

\begin{abstract}
This paper investigates sources of error for a vector velocity volume flow estimator. Quantification of the estimator's accuracy is performed theoretically and investigated in vivo. Womersley's model for pulsatile flow is used to simulate velocity profiles and calculate volume flow errors in cases of elliptical vessels and not placing the transducer at the vessel center. Simulations show, i.e., that volume flow is underestimated with $5 \%$, when the transducer is placed $15 \%$ from the vessel center. Twenty patients with arteriovenous fistulas for hemodialysis are scanned in a clinical study. A BK Medical UltraView 800 ultrasound scanner with a $9 \mathrm{MHz}$ linear array transducer is used to obtain Vector Flow Imaging sequences of a superficial part of the fistulas. Cross-sectional diameters of each fistula are measured on B-mode images by rotating the scan plane 90 degrees. The major axis of the fistulas was on average $8.6 \%$ larger than the minor axis, so elliptic dimensions should be taken into account in volume flow estimation. The ultrasound beam was on average $1.5 \pm 0.8 \mathrm{~mm}$ off-axis, corresponding to $28.5 \pm 11.3$ $\%$ of the major semi-axis of a fistula, and this could result in $15 \%$ underestimated volume flow according to the simulation. Volume flow estimates were corrected for the beam being offaxis, but was not able to significantly decrease the error relative to measurements with the reference method.
\end{abstract}

\section{INTRODUCTION}

Quantifying blood flow to organs is desirable for evaluating the pathological state of the vascular system, i.e., in the carotid artery or at arteriovenous fistulas in dialysis [1]. Among the simplest methods for estimating volume flow non-invasively is the single-point Doppler ultrasound method. The peak velocity is estimated at one location along the presumed centerline of a vessel, and by assuming steady flow and a perfect parabolic velocity profile, the volume flow is calculated based on a circular cross-sectional area of the vessel. A more accurate method is to estimate velocities at several points along the whole vessel diameter, since the actual velocity profile, rather than the assumed one, can be included in the estimator [2], [3]. The method resulted in biases of $5 \%$ for constant flow in a phantom and when using conventional Doppler ultrasound for velocity estimation [3]. Doppler ultrasound estimates the velocity component along the ultrasound beam and a major limitation of the methods is, therefore, associated with determining the correct beam-to-flow angle, which introduces error in volume flow estimation. Furthermore, conventional Doppler ultrasound is challenging for estimation of flow in superficial vessels where flow is nearly transverse to the ultrasound beam.
Several methods have been proposed to remedy the angle dependency problem and extend the conventional ultrasound velocity estimate to vector estimates. Transverse Oscillation (TO) is a method capable of estimating the axial and lateral velocity components independent of each other [4]. By integrating the velocity field obtained from $\mathrm{TO}$ vector velocities over a circular cross-section of a vessel, volume flow estimation has been validated in vivo in the right common carotid artery and in arteriovenous fistulas [5], [6].

However, in the clinical application of the technique several challenges have been identified, and the sensitivity to deviations from the estimator assumptions should be quantified. The purpose of this paper is to identify the error sources for a vector velocity volume flow estimator and to study their effects on the accuracy of volume flow estimation. This is investigated theoretically and in vivo.

\section{Materials And Methods}

A volume flow estimator using vector velocities acquired with the TO approach is presented in this section along with the investigated error sources. Methods for the theoretical and experimental procedures are also presented.

\section{A. Volume Flow Estimation and Sources of Error}

TO is an angle-independent method for vector velocity estimation within the ultrasound scan plane. By introducing a lateral oscillation in the pulse-echo field along with the conventional axial oscillation, the received signals become sensitive to both an axial and lateral motion in the field. The transmitted field is weakly focused, and the lateral oscillating field is created in the receive beamforming by changing the apodization function to contain two separated peaks. The axial velocity is estimated as in conventional Doppler ultrasound, while a special autocorrelation estimator is used for the transverse velocity component. The resulting $2 \mathrm{D}$ vector velocity estimate represents the velocity magnitude and direction of flow at a specific point, and the estimated velocity magnitudes at locations along a vessel diameter can be used for volume flow calculation. The volume flow $Q$ of a fluid crossing a circular surface is estimated as

$$
Q=\pi \cdot \Delta g^{2} \sum_{n=-N / 2}^{N / 2} v_{n} \cdot|n|
$$




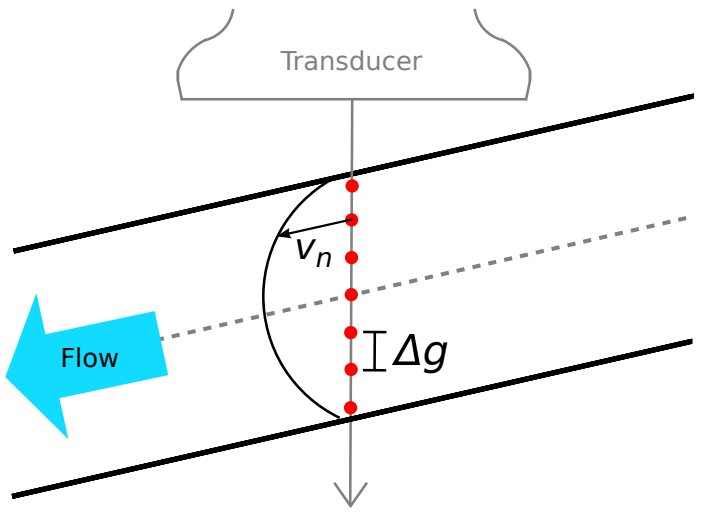

Fig. 1. Longitudinal view of a vessel and velocity estimation at points along the whole vessel diameter. The $n$th vector velocity sample is $v_{n}$ and the distance between two samples is $\Delta g$.
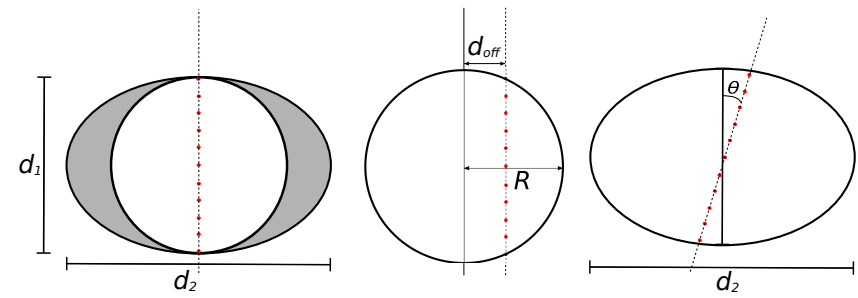

Fig. 2. Sources of error in volume flow estimation: elliptic cross-section (left), beam off-axis (middle) and beam steering in an elliptic vessel (right).

which is a rotation of each vector velocity sample $v_{n}$ around the symmetry axis at the vessel center [3]. The finite number of velocity samples inside the vessel is $N$ and the distance between two samples is $\Delta g$. It is assumed that flow is axisymmetric, the cross-sectional area of the vessel is circular, and that the velocity sampling is along a diameter of the vessel. A geometry of the methodology is shown in Fig. 1. When the beam is swept over a section of the vessel to give a VFI frame, the mean volume flow in the frame is found by averaging the estimates at all lateral positions.

This paper investigates three sources of error in volume flow estimation. An illustration of the errors is shown in Fig. 2. A problem with superficial vessels is that they are easily compressed under the weight of a transducer, which changes the cross-sectional blood vessel area from a circular to elliptical geometry. Another problem is that the ultrasound beam is assumed to intersect the middle of the vessel, but it can be challenging for the examiner to place the transducer at the vessel center. This results in sampling off-axis. Furthermore, the effect of steering the ultrasound beam in a direction that is not along one of the axes of an elliptic vessel should be studied. These three issues introduce error in volume flow estimation, and the error will be quantified in the following.

\section{B. Simulations}

To investigate the effect of beam-vessel intersection, a theoretical investigation was performed with Womersley's model for pulsatile flow [7]. Womersley's model incorporates the pulsatile behaviour of blood flow and creates more realistic physiological waveforms than a parabolic profile. The flow pattern is decomposed into sinusoidal components and added to attain the velocity profile in time and space.

Velocity profiles were created for a number of time steps throughout a cardiac cycle to mimic flow in the carotid artery. The mean velocity of the flow was $0.15 \mathrm{~m} / \mathrm{s}$, the heart rate was 62 beats/min and the Womersley's number was 1.05. The volume flow was calculated from (1) for a number of time steps throughout a cardiac cycle by using the generated velocity samples and $\Delta g$. The spatial average velocity was also calculated and multiplied by the cross-sectional area to find the volume flow for reference.

\section{Experimental Methods}

The effects of vessel ellipticity and beam-vessel intersection on volume flow were investigated for twenty patients with arteriovenous fistulas for hemodialysis.

Scannings were performed with an UltraView 800 ultrasound scanner (BK Medical, Herlev, Denmark) and a $9 \mathrm{MHz}$ linear array transducer (8670, BK Medical). Initially, each patient was scanned longitudinally and transversely directly on the fistula for orientation purposes and to measure two perpendicular diameters of the fistula. The transducer was then rotated $90^{\circ}$ back to record blood flow longitudinally. The transducer was placed where the fistula had its widest diameter and data were recorded over a period of $15 \mathrm{~s}$. The beam-to-flow angle was approximately $90^{\circ}$ and the scans were performed just prior to dialysis. Details of the scanning procedure is described in [5].

From each recorded VFI frame, volume flow was calculated off-line as described in Section II-A, and thereby the average volume flow during a scan sequence of $15 \mathrm{~s}$ was calculated.

Ultrasound Dilution Technique (UDT) is the reference method for measuring volume flow in arteriovenous fistulas and was measured with a Transonic HD03 Flow-QC Hemodialysis Monitor in this study for volume flow comparison [8].

Cross-sectional diameters of a blood vessel were determined by measuring two perpendicular diameters on a B-mode image. The actual vessel diameter along the ultrasound beam was also estimated from VFI data in a scan sequence. The scanner has a build-in blood-tissue discrimination and sets the velocity to zero outside the flow region. Thus, the vessel width $d_{\text {scanner }}$ was found from the number of samples inside the flow region and $\Delta g$.

Volume flow measurements were analysed using a two-way ANOVA with a null hypothesis of equal means between two methods. Patients were used as blocking and the significance level was 0.05 .

\section{Simulation Results}

\section{A. Elliptic Cross-section}

Calculating volume flow through an elliptic and circular cross-section can be performed by multiplying each velocity estimate $v_{n}$ with either the area of a circular semi-annulus $A_{c_{n}}$ 


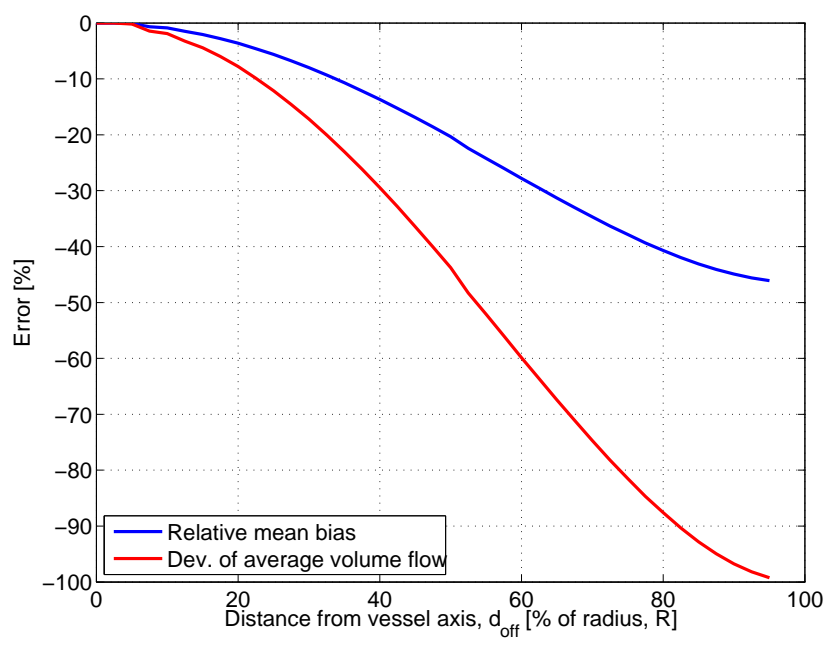

Fig. 3. Beam being off the vessel axis. The distance from the center of the vessel that the beam is off axis, $d_{o f f}$, is expressed as a percentage of radius, $R$. The blue graph is mean relative bias scaled with peak volume flow and the red graph is deviation of volume flow averaged over a cardiac cycle.

or an elliptic semi-annulus $A_{e_{n}}$, respectively,

$\frac{Q_{\text {ellipse }}}{Q_{\text {circ }}}=\frac{\sum_{n=-N / 2}^{N / 2} A_{e_{n}} v_{n}}{\sum_{n=-N / 2}^{N / 2} A_{c_{n}} v_{n}}=\frac{\sum_{n=-N / 2}^{N / 2} \frac{1}{2} \pi\left[d_{1_{n}} d_{2_{n}}-d_{1_{n-1}} d_{2_{n-1}}\right] v_{n}}{\sum_{n=-N / 2}^{N / 2} \pi\left[d_{1_{n}}^{2}-d_{1_{n-1}}^{2}\right] v_{n}}$

$$
\begin{gathered}
=\frac{\sum_{n=-N / 2}^{N / 2}\left[\left(n^{2} \Delta h \Delta g\right)-(n-1)^{2} \Delta h \Delta g\right] v_{n}}{\sum_{n=-N / 2}^{N / 2}\left[(n \Delta g)^{2}-((n-1) \Delta g)^{2}\right] v_{n}} \\
=\frac{\Delta h \cdot \sum_{n=-N / 2}^{N / 2}[2 n-1] v_{n}}{\Delta g \cdot \sum_{n=-N / 2}^{N / 2}[2 n-1] v_{n}}=\frac{d_{2}}{d_{1}},
\end{gathered}
$$

where $d_{1}$ is the vessel diameter along the ultrasound beam, $d_{2}$ is the diameter in the elevation plane and $\Delta h=d_{2} / N$. The derivation shows that if it is assumed that the vessel has a circular cross-section with diameter $d_{2}$, the error in volume flow estimation is $\left(d_{2} / d_{1}\right)-1$, if the true cross-section is elliptic.

\section{B. Beam Off-axis}

A full velocity distribution inside a circular vessel was obtained by rotating a simulated velocity half-profile symmetrically around the center axis. By sampling the full velocity distribution along a line off the vessel diameter, volume flow was calculated from the velocity samples by assuming a circular distribution of the samples and using (1). The volume flow was then compared to the true volume flow, and the estimation error is shown in Fig. 3. The off-axis distance $d_{o f f}$ from the vessel center is expressed as a percentage of vessel radius $R$, and the blue graph represents the mean relative bias scaled with peak volume flow, while the red graph is the deviation of average flow in a cardiac cycle. Both graphs have the characteristic shape of a sigmoid curve.

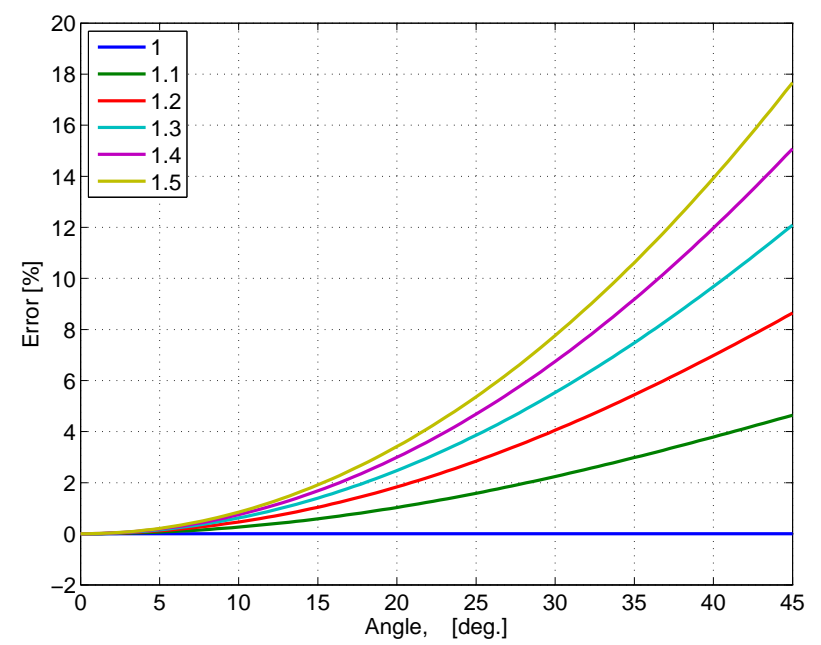

Fig. 4. Volume flow error when the ultrasound beam is steered from 0 to $45^{\circ}$ in a vessel with elliptic cross-section. Each graph is for a specific elliptic geometry, $d_{2} / d_{1}$.

\section{Beam Steering in Elliptical Vessels}

For volume flow estimation in an elliptical vessel, it is assumed that the ultrasound beam is steered in a direction along one of the diameters. The effect of steering in another direction is presented in this section. By keeping one of the diameters fixed (i.e., $d_{2}$ on a B-mode image) and measuring $d_{1}$ as the width of the actual velocity profile along the direction of the ultrasound beam, volume flow error was calculated for the beam steered from 0 to $45^{\circ}$. The result is shown in Fig. 4 for simulated velocity profiles, and each graph in the figure represent an elliptic geometry.

\section{EXPERIMENTAL RESUlTS}

\section{A. Elliptic Cross-section}

A B-mode image of a cross-section of an arteriovenous fistula and its diameters is shown in Fig. 5. Measuring $d_{2} / d_{1}$ for all 20 patients gives an indication of how elliptic the crosssectional fistulas were. The mean \pm one std. of $d_{2} / d_{1}$ was $1.086 \pm 0.105$, so that $d_{2}$ on average was $8.6 \%$ larger than $d_{1}$. To avoid underestimation of volume flow, the dimensions of an elliptic cross-section rather than circular should therefore be taken into account.

\section{B. Beam Off-axis}

To investigate beam-vessel intersection in a clinical study, a calculation of the actual vessel diameter, $d_{\text {scanner }}$, was performed. This diameter was compared to the diameter $d_{1}$ on the B-mode image for each patient. For half of the patients, $d_{\text {scanner }}$ was larger than $d_{1}$ on the B-mode image, indicating that the transducer was moved. For the rest of the patients, the beam was off-axis and an off-axis distance relative to $d_{2}$ was calculated based on the fistula dimensions. By averaging the off-axis distances over all patients, the mean \pm one std. is $0.15 \pm 0.08 \mathrm{~cm}$. This corresponds to $28.5 \pm 11.3 \%$ relative 

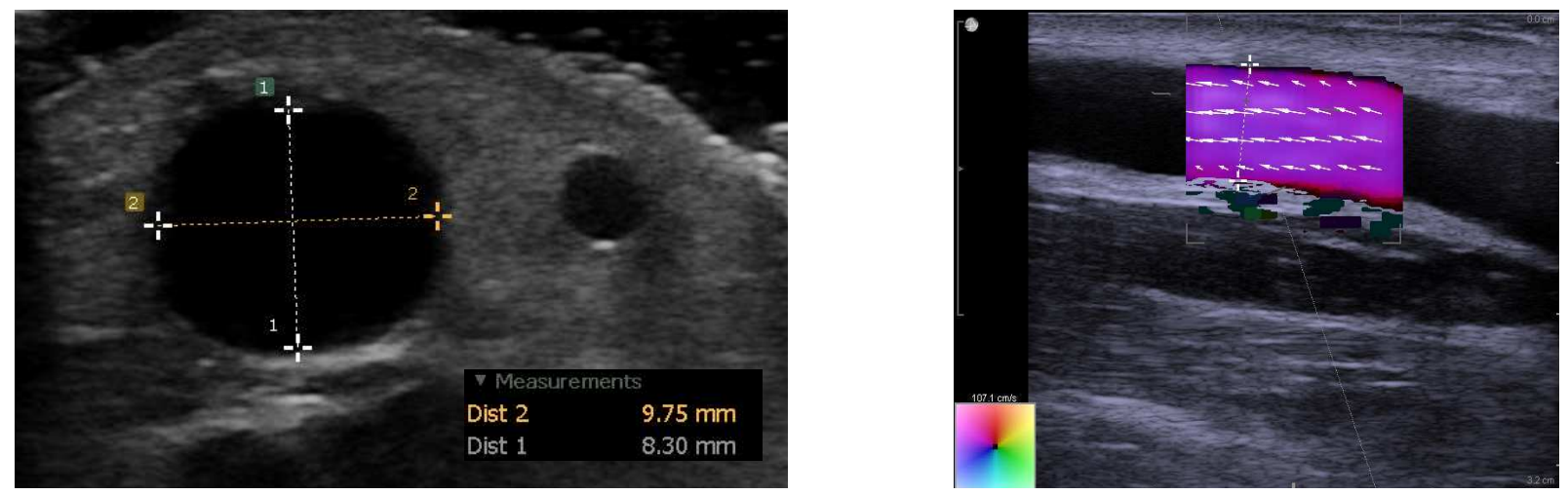

Fig. 5. Example of a B-mode image of an arteriovenous fistula for measurements of the cross-sectional diameters $d_{1}$ and $d_{2}$ (left) and a longitudinal VFI scan (right). The arrows indicate velocity direction and magnitude.

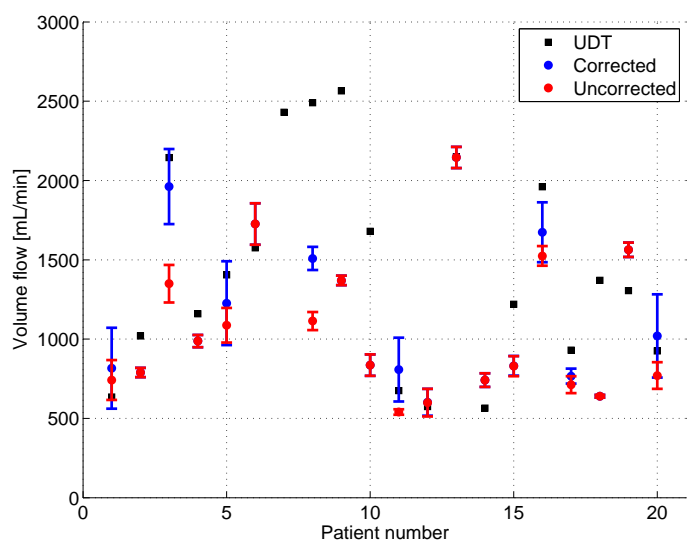

Fig. 6. Volume flow estimates corrected for the beam being off-axis (blue), uncorrected estimates (red), and UDT measurements (black). Each bar represents the mean \pm 1 std.

to $d_{2}$. The results indicate that even though the scans were performed carefully by an experienced medical doctor, it was difficult to scan with the beam in the center of a vessel.

For patients where the beam was off-axis $\left(d_{\text {scanner }}<d_{1}\right)$, the volume flow estimates were corrected. The off-axis distance for each patient and correction factors based on the simulated results in Fig. 3 (red graph) were used to calculate corrected volume flow estimates. Fig. 6 shows the results for each patient.

The uncorrected volume flow estimates deviate with a mean \pm one std. of $26.3 \pm 16 \%$ compared to the UDT measurements. The deviation of volume flow after correction of the beam being off-axis is $23 \pm 15 \%$. Therefore, the correction is able to decrease the error relative to UDT, however, not significantly $(p=0.92)$.

\section{Discussion AND CONCLUSION}

The effects of vessel ellipticity and not placing the ultrasound transducer at the vessel center have been presented for vector velocity volume flow estimation. The errors have been quantified theoretically and studied in vivo. It has been shown that the dimensions of elliptic vessels and beam-vessel intersection should be taken into account to avoid volume flow underestimation. When the beam is, i.e., $15 \%$ from the vessel center, volume flow is underestimated with $5 \%$. The beam was on average $28.5 \pm 11.3 \%$ off-axis for the clinical study and could lead to $15 \%$ underestimated volume flow according to the simulation. A correction for the beam being off-axis was not able to significantly decrease the error, but difficulties with UDT comparison should be taken into account [5].

A $90^{\circ}$ rotation of the transducer is needed for measuring elliptic cross-sectional diameters, however, the transducer rotation can lead to the beam being off-axis. It is therefore recommended to use cross-sectional B-mode scans as guidance for beam-vessel intersection.

\section{ACKNOWLEDGEMENT}

This work was supported by grant 82-2012-4 from the Danish National Advanced Technology Foundation and by BK Medical.

\section{REFERENCES}

[1] P. Wiese and B. Nonnast-Daniel, "Colour doppler ultrasound in dialysis access," Nephrology Dialysis Transplantation, vol. 19, no. 8, pp. 19561963, 2004.

[2] P. A. Picot and P. M. Embree, "Quantitative volume flow estimation using velocity profiles," IEEE Trans. Ultrason., Ferroelec., Freq. Contr., vol. 41, pp. 340-345, 1994.

[3] S. Ricci, M. Cinthio, Å. Ahlgren, and P. Tortoli, "Accuracy and reproducibility of a novel dynamic volume flow measurement method," Ultrasound Med. Biol., vol. 39, no. 10, pp. 1903-1914, 2013.

[4] J. A. Jensen and P. Munk, "A New Method for Estimation of Velocity Vectors," IEEE Trans. Ultrason., Ferroelec., Freq. Contr., vol. 45, pp. 837-851, 1998

[5] P. M. Hansen, J. B. Olesen, M. J. Pihl, T. Lange, S. Heerwagen, M. M. Pedersen, M. Rix, L. Lønn, J. A. Jensen, and M. B. Nielsen, "Volume flow in arteriovenous fistulas using vector velocity ultrasound," Ultrasound Med. Biol., p. Accepted for publication, 2014.

[6] K. L. Hansen, J. Udesen, C. Thomsen, J. A. Jensen, and M. B. Nielsen, "In vivo validation of a blood vector velocity estimator with MR angiography," IEEE Trans. Ultrason., Ferroelec., Freq. Contr., vol. 56, no. 1, pp. 91-100, 2009.

[7] J. R. Womersley, "Oscillatory motion of a viscous liquid in a thin-walled elastic tube. I: The linear approximation for long waves," Phil. Mag., vol. 46, pp. 199-221, 1955.

[8] N. M. Krivitski, "Theory and validation of access flow measurement by dilution technique during hemodialysis," Kidney International, vol. 48, no. 1, pp. 244-250, jul 1995. 